\section{Dengue Virus Type 3 Infection in Traveler Returning from West Africa}

To the Editor: GeoSentinel, the global surveillance program of the International Society of Travel Medicine, recently reported that returning travelers may serve as sentinels for local outbreaks of dengue fever in tropical areas to which it is endemic (1). We investigated a case of dengue virus (DENV) type 3 (DENV-3) infection in a traveler returning to France from West Africa, which provided evidence for DENV-3 circulation in Côte d'Ivoire.

A 53-year-old French expatriate living in Abidjan, the economic capital of Côte d'Ivoire, arrived in France on July 17,2008 , and was hospitalized 4 days later with fever of $40^{\circ} \mathrm{C}$, headache, asthenia, anorexia, chills, diffuse arthralgia, and myalgia. Results of a physical examination were normal except for a diffuse nonpetechial macular rash and moderate hepatosplenomegaly. A tourniquet test was not performed. At admission, platelet count was 103 cells $/ \mathrm{mm}^{3}$ and leukocyte count was $2,410 \mathrm{cells} / \mathrm{mm}^{3}$. Thin and thick blood smears and results of the immunochromatographic test (Binax NOW malaria tests; Binax, Portland, ME, USA) were negative. The patient recovered without sequelae and was discharged 6 days after admission.

At admission, chikungunya virus-specific immunoglobulin (Ig) G and $\operatorname{IgM}$ were not detected by indirect immunofluorescence tests (2). IgG, but not IgM, specific for DENV was detected by an immunochromatic test (Panbio Dengue Duo Cassette; Biotrin, Lyon, France) and confirmed by ELISA (PanBio dengue duo test). However, DENV RNA was demonstrated in serum by using 4 reversetranscription-PCR (RT-PCR)-based assays: a positive result in a flavivirus universal assay (3), a positive result in a DENV-1-4 real-time RT-PCR (4), a positive result in a DENV-3-specific RT-PCR, and a negative result in a specific RT-PCR for DENV-1, -2, and -4 (4). The concomitant finding of DENV RNA and IgG against DENV suggests the patient had dengue infection before this episode.

DENV-3 viremia was confirmed by sequencing a 547-nt region of the envelope gene (GenBank accession no. FJ587232, nt 852-1398 referring to the H87 DENV-3 prototype strain). Our sequence aligned with homologous DENV-3 sequences retrieved from GenBank and used for phylogenetic analysis (Figure). Our patient, in whom classic dengue fever was diagnosed, was infected with a strain that belonged to genotype III most closely related to strains isolated in Singa-

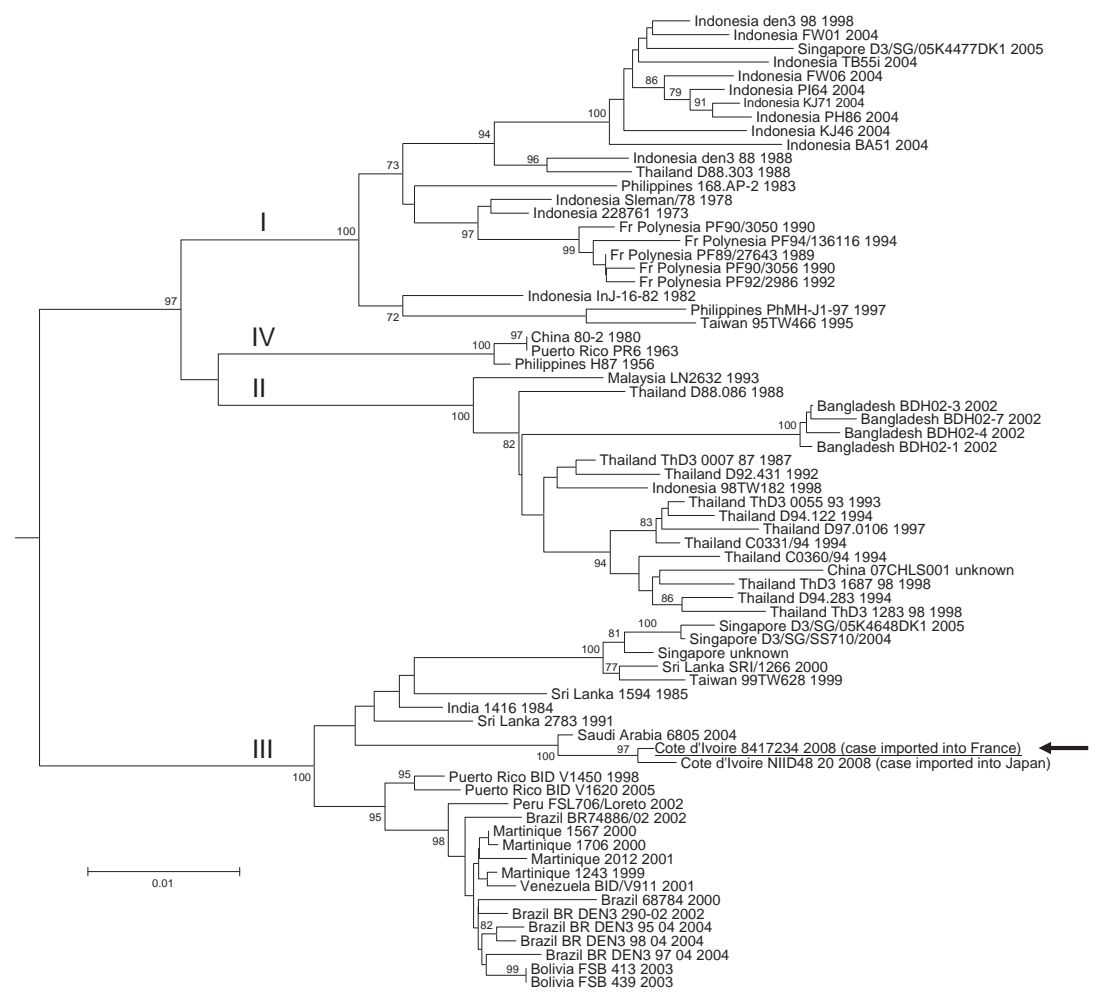

Figure. Phylogenetic analysis of selected dengue virus type 3 (DENV-3) sequences. The main genotypes are indicated using roman numerals at the node of the lineage. Sequence identification is as follows: country of origin, strain name, year of isolation/detection. The sequence determined in our study is underlined and designated by an arrow. Phylogenetic studies were conducted by using MEGA version 2.1 (5). Genetic distances were calculated with the Kimura 2-parameter method at the nucleotide level. Phylogenetic trees were constructed using the neighbor-joining method. The robustness of the nodes was tested by 500 bootstrap replications. The tree was rooted with DENV-1, DENV-2, and DENV-4 sequences. Scale bar indicates nucleotide substitutions per site. 
DENV-3 strains recently caused unexpected outbreaks of dengue hemorrhagic fever in Sri Lanka, East Africa, and Latin America (9).

The case presented here demonstrates that epidemics may be undetected or unidentified until diagnosis is assessed in another country from a returning infected visitor, thus drawing attention to an unidentified potential epidemic situation. This situation can be unraveled by a clinician who considers geographic factors in the diagnostic workup and has access to and uses appropriate laboratory capacity to diagnose imported infections. At the time dengue was diagnosed in this patient, cases of yellow fever were reported in the same location of Côte d'Ivoire (Abidjan) (5), illustrating concomitant circulation of 2 viruses in which dengue may have remained undetected in the absence of a laboratory-confirmed case in the traveler's home country. Therefore, this case reinforces the utility of travelers as sentinels for infectious diseases as previously reported (10). Our findings reiterate the need for technologic transfer of PCR-based direct diagnostics to reference centers in areas where emergence is likely. These efforts also should embrace serology and encourage close collaboration with world reference centers for confirmation and characterization (10).

\section{Acknowledgments}

We thank David Freedman, EuroTravNet members, and the reviewers for helpful comments and discussion.

\section{Laetitia Ninove, Philippe Parola, Cécile Baronti, Xavier De Lamballerie, Philippe Gautret, Barbara Doudier, and Rémi N. Charrel}

Author affiliation: Fédération de Microbiologie Clinique Assistance Publique Hôpitaux de Marseille, Marseille, France

DOI: 10.3201/eid1511.081736

\section{References}

1. Schwartz E, Weld LH, Wilder-Smith A, von Sonnenburg F, Keystone JS, Kain $\mathrm{KC}$, et al. GeoSentinel Surveillance Network. Seasonality, annual trends, and characteristics of dengue among ill returned travelers, 1997-2006. Emerg Infect Dis. 2008;14:1081-8. DOI: 10.3201/ eid1407.071412

2. Fulhorst CF, Monroe MC, Salas RA, Duno G, Utrera A, Ksiazek TG, et al. Isolation, characterization and geographic distribution of Cano Delgadito virus, a newly discovered South American hantavirus (family Bunyaviridae). Virus Res. 1997;51:159-71. DOI: 10.1016/S01681702(97)00091-9

3. Moureau G, Temmam S, Gonzalez JP, Charrel RN, Grard G, de Lamballerie X. A real-time RT-PCR method for the universal detection and identification of flaviviruses. Vector Borne Zoonotic Dis. 2007;7:46777. DOI: 10.1089/vbz.2007.0206

4. Leparc-Goffart I, Baragatti M, Temman S, Tuiskunen A, Moureau G, Charrel R, et al Development and validation of real time one-step reverse transcription-PCR for the detection and typing of dengue viruses. $\mathrm{J}$ Clin Virol. 2009;45:61-6.

5. Kumar S, Tamura K, Jakobsen IB, Nei M. MEGA2: Molecular Evolutionary Genetics Analysis software. Tempe (AZ): Arizona State University; 2001.

6. Dengue in Africa: emergence of DENV-3, Côte d'Ivoire, 2008. Wkly Epidemiol Rec. 2009;84:85-8.

7. Durand JP, Vallée L, de Pina JJ, Tolou H. Isolation of a dengue type 1 virus from a soldier in West Africa (Côte d'Ivoire). Emerg Infect Dis. 2000;6:83-4. DOI: 10.3201/eid0602.000211

8. Leroy EM, Nkogue D, Ollomo B, NzeNkogue C, Becquart P, Grard G, et al. Concurrent chikungunya and dengue virus infections during simultaneous outbreaks, Gabon, 2007. Emerg Infect Dis. 2009;15:591-3. DOI: 10.3201/ eid1504.080664

9. Messer WB, Gubler DJ, Harris E, Sivananthan K, de Silva AM. Emergence and global spread of a dengue serotype 3, subtype III virus. Emerg Infect Dis. 2003;9:800-9.

10. Freedman DO, Weld LH, Kozarsky PE, Fisk T, Robins R, von Sonnenburg F, et al. Spectrum of disease and relation to place of exposure among ill returned travelers. N Engl J Med. 2006;354:119-30. DOI: 10.1056/NEJMoa051331

Address for correspondence: Rémi N. Charrel, Université de la Mediterranee-Unite des Virus Emergents, 27 bd Jean Moulin, Marseille 13005 , France; email: rnc-virophdm@gulliver.fr
Low Immunity to Measles and Rubella among Female Guest Workers, Northern Mariana Islands

To the Editor: The Commonwealth of the Northern Mariana Islands (CNMI), a group of northern Pacific islands in political union with the United States, was exempt from US labor laws until 2007. This exemption attracted business opportunities, which led to a high demand for guest workers. The Centers for Disease Control and Prevention advises the US Citizenship and Immigration Services of vaccination requirements for those applying for immigration and work visas before the applications are approved (1). Since 1996, all applicants born after 1957 and >12 months of age have been required to provide evidence of completed vaccination against, or of immunity to, measles, mumps, and rubella viruses. Those unable to provide such evidence must receive at least 1 dose of the vaccines recommended by the US Advisory Committee on Immunization Practices before visa approval. The Committee also advises applicants to receive additional doses of the required vaccines after arrival in the Mariana Islands. We aimed to determine the proportion of CNMI guest workers who were immune to measles and rubella by testing a convenience sample of serum collected during September and October 2006. However, procedures for validating the vaccination status for our sample population are unknown. Given our results, it appears that validation procedures of immunity status in guest workers or immigrants to the United States were suboptimal at the time of this study.

Serum samples from 210 female workers from 17 through 51 years of age were collected opportunistically when, as a requirement for annual con- 